Revista Ibero- Americana de Humanidades, Ciências e Educação- REASE $\quad$ open $C$ access

doi.org/ 10.51891/rease.v7iro.2848

\title{
O ENFERMEIRO FRENTE À CLASSIFICAÇÃO DE RISCO EM URGÊNCIA E EMERGÊNCIA: UMA REVISÃO INTEGRATIVA DE LITERATURA
}

\author{
Juan Fernando Damacena da Silva ${ }^{1}$ \\ Jaine Cristina Pancera ${ }^{2}$ \\ Fátima Regina Cividini ${ }^{3}$
}

RESUMO: Os serviços de emergência e urgência são as portas de entrada de muitos que procuram atendimento de diversos tipos de enfermidades. Com o surgimento da classificação de risco, cuja finalidade é prestar uma maior qualidade e presteza no atendimento seguindo as necessidades de cada paciente. Deste modo, a assistência do enfermeiro se destaca, em estar na linha de frente como encarregado pela aplicação do protocolo de classificação de risco. Tratase de uma pesquisa qualitativa do tipo Revisão integrativa de Literatura, cuja busca foi realizada na Scientific Eletronic Library Online (SciELO), Bireme, LILACS e bases encontradas no Portal de Periódicos Capes, entre 2008 e 2019. Foram utilizados como critérios de inclusão: artigos disponíveis em português, publicados entre 2008 e 2019, que debatem temas relacionados ao enfermeiro de classificação de risco. Na literatura, demonstra-se o papel do enfermeiro na classificação de risco, realizando o acolhimento e triagem do paciente e classificando de acordo com as necessidades de cada. Conclui-se que, é essencial destacar, que 2461 apesar dos desafios achados a classificação de risco se mostra um meio preciso para a técnica de trabalho eficiente, e de importante relevância para classificação.

Palavras-chave: Classificação de Risco. Enfermeiro. Emergência e Urgência. Protocolo de Manchester.

ABSTRACT: Emergency and urgent services are the gateways for many who seek care for different types of illnesses. With the emergence of risk classification, whose purpose is to provide greater quality and promptness in care following the needs of each patient. In this way, the nurse's assistance stands out, in being in the front line as in charge of the application of the risk classification protocol. It is a qualitative research of the integrative Literature Review type, searched for in Scientific Electronic Library Online (SciELO), Bireme, LILACS and databases found in the Capes Journal Portal, between 2008 and 2019. The inclusion criteria were: articles available in Portuguese, published between 2008 and 2019, which discuss

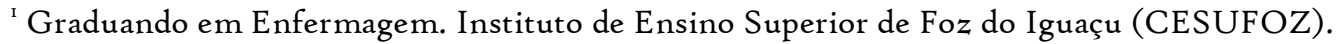

${ }^{2}$ Graduando em Enfermagem. Instituto de Ensino Superior de Foz do Iguaçu (CESUFOZ).

${ }^{3}$ Enfermeira. Mestre e Doutoranda em Sociedade, Culturas e Fronteiras (UNIOESTE-FOZ). Docente do curso de Enfermagem pelo Instituto de Ensino Superior de Foz do Iguaçu (CESUFOZ). ORCID: 0oo3-4129-7915. Email para contato: fatima.cividini@docente.suafaculdade.com.br
} 
topics related to the risk classification nurse. In the literature, the role of nurses in risk classification is demonstrated, carrying out the reception and screening of the patient and classifying them according to the needs of each. It is concluded that, it is essential to emphasize that, despite the challenges found, risk classification is a precise means for an efficient work technique, and of important relevance for classification.

Keywords: Risk rating. Nurse. Emergency and Urgency. Manchester Protocol.

\section{INTRODUÇÃO}

Os serviços de emergência lidam com a elevação crescente na busca por seus serviços, acolhendo pacientes de urgência, pacientes com quadros percebidos como urgências, pacientes que não têm acesso à atenção primária e especializada e as urgências sociais. Todas essas demandas, consequentemente, sobrecarregam os serviços de emergência, o que leva a uma diminuição da qualidade da assistência prestada à população (BRASIL, 2006).

$\mathrm{Na}$ tentativa de solucionar o problema e atender as demandas conforme a urgência do caso, o Ministério da Saúde através da Portaria no 2048 de 2004 sugere a implantação nas unidades de urgência e emergência o acolhimento e a triagem classificatória de risco. Segundo ela, o trabalho deve ser realizado por profissional de nível superior que estejam capacitados para utilizar os protocolos e avaliar o grau de urgência dos pacientes atendidos, e priorizar o paciente de acordo com suas necessidades. (BRASIL, 2004)

Os sistemas de triagem, portanto, têm como prioridade organizar o fluxo de pacientes que chegam em busca de atendimento em serviços de urgência, apontando os que necessitam de prioridade no atendimento e reconhecendo aqueles que podem aguardar o atendimento em segurança pela ordem de chegada. Essa avaliação da classificação de risco é realizada por um enfermeiro (ACOSTA; DURO; LIMA, 2012).

O protocolo de Manchester é o mais comumente utilizado no Brasil, estruturado por fluxogramas que representam as queixas apresentadas por quem busca os serviços de emergência. $O$ paciente pode ser classificado em um dos cinco diferentes níveis de prioridades: emergência com a cor vermelha com o tempo de espera de zero minutos, muito urgente de cor laranja com o tempo de espera de até dez minutos, urgente de cor amarelo com o tempo máximo de espera de até sessenta minutos, pouco urgente de cor verde com tempo de 
espera de até cento e vinte minutos e não urgente de cor azul com o tempo de espera de até duzentos e quarenta minutos (SOUZA et al, 2013)

No entanto, acolher e classificar o risco trazido por um paciente é um momento de atenção por parte do profissional enfermeiro que atua na sala de triagem e classificação de risco. Contudo, a partir das ações aplicadas a cada paciente que chega ao setor de classificação, faz-se necessário que gestores e profissionais de saúde valorizem e favoreçam a educação em serviço, mobilizando seus funcionários para a formação de grupos de estudos locais de forma a refletir sobre a prática da educação com base na necessidade de organizá-la em razão dos objetivos que se quer alcançar (SILVA; BARROS; TORRES, 20I2).

Assim, o enfermeiro torna-se o profissional fundamental na triagem, primeiro atendimento e a classificação de risco para que não ocorra erros, pois no momento emergencial exige rapidez. Desta forma, o enfermeiro necessita estar amparado por sua equipe para atendimento das intercorrências que surgem no setor de emergência baseado em protocolos validados e baseado em evidências. Para isso, é necessário que ele avalie e faça questionamentos necessários para que o paciente seja classificado de acordo com a sua necessidade (SILVA; BARROS; TORRES, 2012; SOUZA et al, 2013)

A partir das reflexões apresentadas, adota-se como objetivo geral conhecer o papel do enfermeiro frente à classificação de risco em urgência e emergência. Diante disso, faz-se a seguinte questão de pesquisa: Qual a atuação do enfermeiro na classificação de risco nos serviços de urgência e emergência?

\section{METODOLOGIA}

O presente estudo será desenvolvido por meio de uma pesquisa bibliográfica, de base qualitativa com natureza descritiva e exploratória, do tipo revisão de literatura, que consiste em na pesquisa como objeto de estudo o "[...] registro disponível, decorrente de pesquisas anteriores [...]" (SEVERINO, 20I6, p.I3I). Este método propõe que os textos são as fontes de estudo e os resultados são as contribuições que cada autor traz para a área acadêmica. (SEVERINO, 2016) 


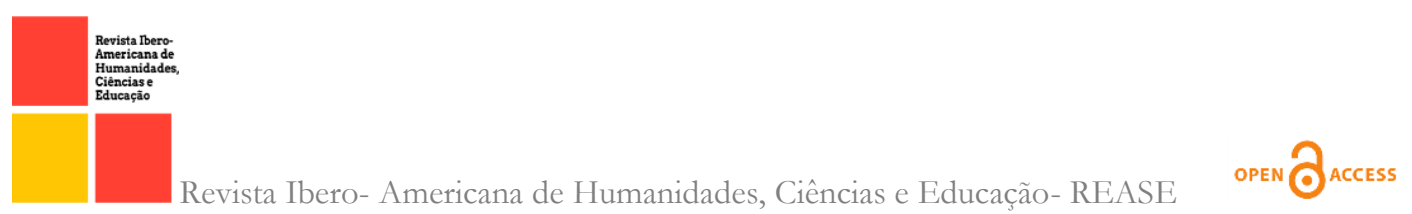

Para tal, realizou-se a busca de dados bibliográficos nas bases de dados Scientific Eletronic Library Online (SciELO), BVS/BIREME, LILACS. Como critérios de inclusão, foram selecionadas publicações em revistas científicas, na língua portuguesa, originais. Utilizaram-se os Descritores em Ciências da Saúde (DeCS): “classificação de risco” ou "protocolo de Manchester" ou "classificação de risco em urgência e emergência" com os operadores booleanos and "enfermagem" and "hospital".

Serão excluídos nesta busca materiais considerados literatura cinzenta: teses, dissertações, artigos de anais de evento, cartas ao editor. Os dados resultarão em informações que responderão à temática proposta. Após a coleta de dados, a análise seguirá de análise do conteúdo, desdobrando-se em etapas de pré-análise, exploração do material, interpretação e categorização conforme os temas que emergiram do material (BARDIN, 2016)

Por ser um estudo de revisão de literatura, foram mencionados os textos conforme autoria. Por ser um estudo que não envolve diretamente seres humanos, não foi necessário submetê-lo ao Comitê de Ética e Pesquisa em Seres Humanos.

\section{RESULTADOS E DISCUSSÃO}

Foram encontrados 22 artigos na busca inicial, dos quais foram utilizados in artigos que constam responderam ao objetivo deste estudo. Os artigos se encontram organizados da seguinte maneira nas bases de dados: Scientific Eletronic Library Online - SciELO (4 artigos), Literatura Latino-Americana e do Caribe em Ciências da Saúde - LILACS (3 artigos) e Bireme (4 artigos). Utilizamos artigos dos seguintes períodos de publicação de 2008 a 2019.

Tabela I - Artigos selecionados de acordo com o ano de publicação, Brasil, 202I.

\begin{tabular}{|ccc|}
\hline Ano de Publicação & Frequência & $\%$ \\
\hline 2008 & o1 & 9, IO \\
2010 & 02 & 18,20 \\
2011 & 02 & I8,20 \\
2013 & o1 & 9, IO \\
\hline
\end{tabular}




\begin{tabular}{|c|c|c|}
\hline 2014 & OI & 9,10 \\
\hline 2016 & 02 & 18,20 \\
\hline 2017 & оI & 9,10 \\
\hline 2019 & оI & 9,IO \\
\hline Total & II & 100 \\
\hline
\end{tabular}

Fonte: Organizado pelos autores (202I)

A partir da leitura dos artigos selecionados, traz-se para esta pesquisa as contribuições dos autores para elucidar a importância do enfermeiro nos serviços de urgência no processo de classificação de risco conforme o Protocolo de Manchester. Para a aplicação desses protocolos, o enfermeiro dentre os profissionais de enfermagem tem sido mencionado para realizar a avaliação e classificar a necessidade daqueles que buscam as unidades de emergência, adquirindo papel de grande importância na hora de fazer a regulação e decidir a prioridade na assistência dos usuários (SALVADOR et al, 2010; DURO; LIMA, 2010; CRUZ et al, 2016; OLIVEIRA et al, 2016; NUNES et al, 2017; QUARESMA; XAVIER; CEZAR-VAZ, 2or9).

O Enfermeiro, conforme os estudos selecionados, tem sido o profissional indicado para avaliar e classificar a gravidade dos que procuram os serviços de emergência, pois, atribui um 2465 grau de risco ao paciente consistindo em um complexo processo de tomada de decisão, possibilitando a priorização do atendimento (DURO; LIMA, 2010; OLIVEIRA et al, 2016 FERREIRA et al, 2016; CRUZ et al, 2016; NUNES et al, 2017; FARIAS; BRASILEIRO, 2018).

Por isso, é importante que o enfermeiro realize a coleta de informações, baseando-se, principalmente, na escuta dos antecedentes clínicos e da queixa principal somada à análise de exame físico, a fim de se identificar os sinais e sintomas, possibilitando o reconhecimento de padrões normais ou alterados e o julgamento de probabilidade do risco (NUNES et al, 2017; QUARESMA; XAVIER; CEZAR-VAZ, 2019). O primeiro contato feito entre o enfermeiro e o paciente tem como finalidade verificar prioridades de assistência à saúde, por meio de um conjunto de observação do mesmo, através de uma visão holística, ou seja, saber ouvir as queixas que o levaram a procurar esse serviço seja elas físicas, psíquicas ou sociais (SALVADOR et al, 2010; DURO; LIMA, 2010; FARIAS; BRASILEIRO, 2018). Assim o 
enfermeiro necessita estar apto para o primeiro atendimento e triagem, conforme Acosta, Duro e Lima (2012):

O enfermeiro deve estar preparado para classificar e, se necessário, reclassificar a prioridade de atendimento do usuário ao longo do período de espera. Para isso, a avaliação do enfermeiro deve ser cíclica, ou seja, requer contínuo planejamento e reavaliações dos usuários (ACOSTA; DURO; LIMA, 2012, p.187)

Neste sentido, é preciso que o enfermeiro tenha tranquilidade, agilidade, capacidade para tomar decisões rápidas, seguras, contínuas e livres de riscos adicionais, de forma a se adaptar, de imediato, a cada situação que se apresente. Para tanto, é necessário estar preparado para a atuação diante das intercorrências, fundamentado em conhecimento técnico e científico (SALVADOR et al, 2oro; FERREIRA et al, 2oi6; QUARESMA; XAVIER; CEZAR-VAZ, 2019).

Desta forma, é importante que o enfermeiro esteja ciente de suas responsabilidades e, a partir de treinamentos e capacitações constantes, possa estar atento ao que é atual na classificação de risco de pacientes graves (SALVADOR et al, 2010), pois "[...] enfermeiros experientes transmitem segurança para a equipe de classificação de risco, já que os profissionais menos experientes os procuram para auxílio e suporte quando têm dúvidas" 2466 (ACOSTA; DURO; LIMA, 2012, p.187)

Ainda é prioridade do enfermeiro, compreender e administrar o mecanismo de categorização do risco de cada indivíduo. Através do uso de um protocolo previamente selecionado como diretriz técnica que embasa teoricamente a sua decisão (QUARESMA; XAVIER; CEZAR-VAZ, 2019). É o enfermeiro quem decide qual informação precisará ser obtida no que diz respeito ao estado de saúde de um cliente, avaliando tanto dados objetivos quanto subjetivos. O protocolo deverá ser a diretriz base de orientação, trazendo experiências concretas e embasadas cientificamente (DURO; LIMA, 2010; ACOSTA; DURO; LIMA, 2012; BELLUCCI; MATSUDA, 2012; OLIVEIRA et al, 2016; FARIAS; BRASILEIRO, 2018).

Nesse sentido, o enfermeiro que realiza essa atividade é muitas vezes o primeiro profissional de saúde que famílias e pacientes veem quando chegam ao serviço. Portanto, é necessário que ele tenha excelentes habilidades de comunicação para ajudar essas pessoas em um momento tão vulnerável e até para orientar o indivíduo e sua família sobre o tipo de 
atendimento necessário e o tempo de espera provável (DURO; LIMA, 2010; SALVADOR et al, 2oro; OLIVEIRA et al, 2or6; QUARESMA; XAVIER; CEZAR-VAZ, 2019).

Ainda, esse profissional deve estar disposto à conversa e ao diálogo, permitindo entender as necessidades de saúde apresentadas pelo usuário, buscando a solução do problema e criando a possibilidade do fortalecimento da rede de atenção, à medida que referência para outros serviços de saúde mais apropriados para cada situação apresentada. Além disso, na visão dos enfermeiros, na classificação de risco o profissional está acolhendo o usuário, ouvindo suas queixas e dando respostas a seus questionamentos. Com isso, o enfermeiro estabelece uma relação empática com o indivíduo, minimizando muitas vezes os sentimentos como a ansiedade, a agressividade ou a impaciência que possam surgir no decorrer do atendimento no serviço (ACOSTA; DURO; LIMA, 20I2; BELLUCCI; MATSUDA, 20ı2; OLIVEIRA et al, 2016; QUARESMA; XAVIER; CEZAR-VAZ, 2019).

\section{CONSIDERAÇÕES FINAIS}

Este estudo teve como objetivo conhecer o papel do enfermeiro frente à classificação de risco em urgência e emergência. A partir dos artigos encontrados, percebe-se a importância do 2467 esclarecimento dos usuários do sistema de saúde sobre a importância da procura por atendimento na atenção primária em casos menos complexos, pois assim evita a sobrecarga no atendimento a emergências. Além disso, é importante que haja práticas de acolhimento em todos os pontos de atendimento.

Diante disso, a demanda por serviços de emergência aumentou nos últimos anos, por isso é fundamental que se tenha uma ampliação do público instalado, competência, organização de ações de regulação, controle e avaliação, trabalho com profissionais para modificar as práticas na relação com os usuários e adoção de estruturas e fluxos de encaminhamento de pacientes entre departamentos.

Assim, o enfermeiro é um elemento fundamental na atividade competente desta ferramenta, visto que a classificação dos riscos é uma responsabilidade particular do enfermeiro. É ele quem faz o exame clínico e crítico das queixas e, a partir desses dados, vai determinar o risco para cada caso. No entanto, para tanto, o enfermeiro deve conhecer todas 
as fases de todo o processo de atitude e, além disso, deve enfatizar a abrangência do ACR, desmistificando a ideia de que é apenas um lugar ou um lugar.

É importante ressaltar que também há campo para a construção da qualidade das ações já implementadas, introduzir outras ações ainda não implementadas e continuar a completar os fluxos e mecanismos de controle social. Espera-se que, a partir desta pesquisa, sejam construídos instrumentos no campo da produção científica, visando o benefício do conhecimento científico daqueles que se interessam por tal temática.

\section{BIBLIOGRAFIA}

ACOSTA, Aline Marques; DURO, Carmen Lucia Mottin; LIMA, Maria Alice Dias da Silva. Atividades do enfermeiro nos sistemas de triagem/classificação de risco nos serviços de urgência: revisão integrativa. Revista Gaúcha de Enfermagem, v. 33, n. 4, p. I8I-190, 2012. Disponível em: https://www.scielo.br/j/rgenf/a/VksMs3vswfTZphYbMJYLTsn/?lang=pt\&format=pdf Acesso em 28 set. 2021.

BARDIN, Laurence. Análise de Conteúdo. São Paulo: Edições 7o, 2016, 279p.

BELLUCCI JÚNIOR, José Aparecido; MATSUDA, Laura Misue. Implantação do acolhimento com classificação de risco em serviço hospitalar de emergência: atuação do enfermeiro. Ciência, Cuidado e Saúde. v.II, n.2, p.396-40I, Abr./Jun. 2012. http://ojs.uem.br/ojs/index.php/CiencCuidSaude/article/viewFile/I4922/pdf Acesso em 30 out. 2021.

BRASIL. Humaniza SUS: acolhimento com avaliação e classificação de risco: um paradigma ético-estético no fazer em saúde. Brasília: Ministério da Saúde, 2004, 48p. Disponível em: https://portaldeboaspraticas.iff.fiocruz.br/wpcontent/uploads/2018/II/acolhimento_com_avaliacao_e_classificacao_de_risco.pdf Acesso em 27 set. 2021.

BRASIL. Política Nacional de Atenção às Urgências. 3. ed. Brasília: Ministério da Saúde, 2006. 
http://bvsms.saude.gov.br/bvs/publicacoes/politica_nacional_atencao_urgencias_zed.pdf. Acesso em 27 set. 2021.

CRUZ, Isabel Pereira et al. Assistência de enfermagem em setores de classificação de risco em urgência e emergência. Ciências Biológicas e da Saúde, v. 3, n. 3, p. 133-150, 2016. Disponível em: https://periodicos.set.edu.br/fitsbiosaude/article/view/340I/2017 Acesso em 25 out. 2021

DURO, Carmen Lucia Mottin; LIMA, Maria Alice Dias da Silva. O papel do enfermeiro nos sistemas de triagem em emergências: análise da literatura. Online Brazilian Jornal Of Nursing, v.9, n.3, p.I-I2, 2010. Disponível em http://www.revenf.bvs.br/scielo.php?script=sci_arttext\&pid=Si67642852010000300015\&lng=pt\&nrm=iso_Acesso em 25 out. 2021.

FARIAS, Marilene Tosta; BRASILEIRO, Marislei de Sousa Espíndula. Os desafios do enfermeiro para atuação no acolhimento e classificação de risco nos serviços de emergência. Revista Científica Multidisciplinar Núcleo do Conhecimento, v. 9, n. 9, 2018. Disponível em: https://www.nucleodoconhecimento.com.br/saude/desafios-do-enfermeiro. Acesso em 24 out. 202I.

FERREIRA, Edinete Bezerra et al. Acolhimento com classificação de risco em serviços de urgência e emergência hospitalar. Revista Interdisciplinar em Saúde, v. 3, n. I, p. I48-ı78, 2016. Disponível em: http://www.reme.org.br/artigo/detalhes/304\#: :text=A\%20miss\%C3\%A30\%20do\%20acolhim ento\%20com,em\%20sofrimento\%20de\%20qualquer\%2onatureza\%22. Acesso em 30 out. 2021.

NUNES, Bruna Xavier et al. Atuação do enfermeiro no acolhimento com classificação de risco: caracterização do atendimento mediante protocolos: uma revisão da literatura. Revista Científica FacMais, n. 3, p. I-2I, 2017. Disponível em: https://revistacientifica.facmais.com.br/wp-content/uploads/2017/o9/ı.-ATUAÇÃO-DOENFERMEIRO-NO-ACOLHIMENTO-COM-CLASSIFICAÇÃO-DE-RISCOCARACTERIZAÇÃO-DO-ATENDIMENTO-MEDIANTE-PROTOCOLOS-UMAREVISÃO-DA-LITERAT.pdf. Acesso em 27 set. 2021. 
OLIVEIRA, João Lucas Campos et al. Atuação do enfermeiro no Acolhimento com Classificação de Risco: um estudo de metassíntese. Ciência, Cuidado e Saúde, v. 15, n. 2, p.374 382, out. 2016. Disponível em: https://www.periodicos.uem.br/ojs/index.php/CiencCuidSaude/article/view/28573 Acesso em 30 out. 202I.

QUARESMA, Adrieli dos Santos; XAVIER, Daiani Modernel; CEZAR-VAZ, Marta Regina. O papel do enfermeiro na classificação de risco nos serviços de urgência e emergência. Revista Enfermagem Atual In Derme, v. 87, n. 25, abr. 2019. Disponível em: https://www.revistaenfermagematual.com.br/index.php/revista/article/view/r5I Acesso em 30 out. 2021.

SALVADOR, Pétala Tuani Candido de Oliveira et al. The pre-hospital care to nursing after an accident with multiple victims: an integrative literature review. Revista de Enfermagem UFPE online, v. 4, n. 3, p. II95-I203, maio 2010. Disponível em: 2470 https://periodicos.ufpe.br/revistas/revistaenfermagem/article/view/6278/5525 . Acesso em: o6 nov. 2021.

SEVERINO, Antônio Joaquim. Metodologia do Trabalho Científico. $24^{\underline{a}}$ ed. São Paulo: Cortez; 2016.317p.

SILVA, Paloma Morais; BARROS, Kelly Pereira; TORRES, Heloísa de Carvalho. Acolhimento com classificação de risco na Atenção Primária: percepção dos profissionais de enfermagem. Revista Mineira de Enfermagem, v. 16, n. 2, p. 225-231, 2012. Disponível em: https://cdn.publisher.gni.link/reme.org.br/pdf/vi6nzaiı.pdf Acesso em 28 set. 202I.

SOUZA, Cristiane Chaves et al. Nursing diagnoses in patients classified as priority level I and II according to the Manchester protocol. Revista da Escola de Enfermagem, v. 47, n. 6, p. 
1318-1324,

2013.

Disponível

em:

https://www.scielo.br/j/reeusp/a/XMCWKfjLmdyy7dnQpwvdZpB/?format=pdf\&lang=pt Acesso em 28 set. 202I. 